\title{
Antimicrobial resistance and prevalence of virulence factor genes in fecal Escherichia coli of Holstein calves fed milk with and without antimicrobials
}

\author{
R. V. V. Pereira, T. M. A. Santos, M. L. Bicalho, L. S. Caixeta, V. S. Machado, and R. C. Bicalho ${ }^{1}$ \\ Department of Population Medicine and Diagnostic Sciences. College of Veterinary Medicine, Cornell University, Ithaca, NY 14853
}

\begin{abstract}
Diarrhea in calves has a significant effect on the dairy industry. A common management practice for preventing or decreasing the effects of such disease in preweaned calves is by the use of antimicrobials in milk or milk replacer. In this study, Escherichia coli antimicrobial resistance in fecal samples collected from calves 2 to $8 \mathrm{~d}$ of age that had or had not received antimicrobials in the milk and that had or had not signs of diarrhea by inspection of fecal consistency were investigated. Specifically, resistance of E. coli isolates to individual antimicrobials, multiresistance patterns, and presence of virulence factors were analyzed. Escherichia coli isolates were tested for susceptibility to 12 antimicrobials by use of a Kirby-Bauer disk diffusion assay. The study was conducted at 3 farms, 1 administering growth-promoting antimicrobials (GPA) in the milk and 2 not using GPA in the milk (NGPA). All isolates were susceptible to ciprofloxacin and cefepime. From the total isolates tested, $84 \%(\mathrm{n}=251)$ were resistant to at least 2 antimicrobials and $81 \%$ ( $\mathrm{n}=$ 251) were resistant to 3 or more antimicrobials. When antimicrobial resistance was compared between GPA and NGPA, it was observed that the GPA group had higher odds of antimicrobial resistance for most of the individual antimicrobials tested. No significant correlation of virulence factors in GPA or NGPA and diarrheic or non-diarrheic (control) fecal samples was found. Of the 32 virulence factors evaluated, 21 were detected in the study population; the incidence of only 1 virulence factor was statistically significant in each of the diarrheic status (diarrheic or non-diarrheic) and treatment status (NGPA or GPA) groups. Phylogenetic analysis based on the nucleotide sequence of the DNA gyrase gene $($ gyr $B)$ from 31 fecal E. coli isolates revealed 3 main clades.
\end{abstract}

Key words: antibiotic resistance, dairy calf, diarrhea, Escherichia coli

Received March 4, 2011.

Accepted May 30, 2011.

${ }^{1}$ Corresponding author: rcb28@cornell.edu

\section{INTRODUCTION}

Diseases of neonatal calves have an important effect on the livestock industry. The United States National Health Monitoring System has estimated the preweaning mortality of dairy calves to be $10.8 \%$ with diarrhea responsible for more than half of those deaths (Wu et al., 2010). A common practice in many dairies is to use subtherapeutic levels of antimicrobials, such as tetracycline and neomycin, added to milk or milk replacers for disease prophylaxis and growth promotion, and to improve feed efficiency (Berge et al., 2005, 2009). In the United States, agriculture could be responsible for as much as $70 \%$ of antimicrobial drug consumption; therefore, agricultural animals are considered an important reservoir and arena for emerging antimicrobial drug resistance (van den Bogaard and Stobberingh, 2000; Khachatryan et al., 2006; Alexander et al., 2010).

Resistance to at least 2 classes of antimicrobial agents in Escherichia coli is presently an ordinary finding in human and veterinary medicine and is having an increasing effect on available therapeutic options (von Baum and Marre, 2005). In humans, E. coli is the predominant causative organism of urinary tract infections (UTI) and one of the most frequently isolated organisms in neonatal meningitis and nosocomial bacteremia (von Baum and Marre, 2005; Hammerum and Heuer, 2009).

The objective of this study was to investigate the use of growth-promoting antibiotics (GPA) in the milk of preweaned calves on the prevalence of antibiotic resistance; GPA-supplemented animals were compared with calves not receiving GPA in the milk (NGPA). Additionally, it was our objective to evaluate the association of 32 virulence factor genes with the incidence of diarrhea in dairy calves and the antimicrobial resistance patterns identified.

\section{MATERIALS AND METHODS}

\section{Farm Management, Study Design, Sample Collection, and Case Definition}

Data were collected from 3 dairy farms located near Ithaca, New York, from August 3 to August 25, 2009. 
These farms were selected because of their long working relationship with the Ambulatory and Production Medicine Clinic at Cornell University (Ithaca, NY). Farm A, milking 3,000 cows, and farm B, milking 800 cows, were selected for collection of fecal samples from calves belonging to the NGPA group. Farm C, milking 4,500 cows, was selected for collection of fecal samples for calves belonging to the GPA group.

For the feeding management, all farm fed calves at birth a total of $4 \mathrm{~L}$ of colostrum and subsequently approximately $3.5 \mathrm{~L}$ of pasteurized waste milk from the hospital pen twice per day to calves until weaning at around 2 mo of age. In farm C, $1.56 \mathrm{~mL}$ of sulfamethazine sodium (Sulmet 12.5\% solution; Fort Dodge Animal Health Inc., Fort Dodge, IA) and $1.04 \mathrm{mg}$ of chlortetracycline hydrochloride (CLTC 100 MR; Phibro Animal Health Corp., Ridgefield Park, NJ) per liter of milk. Additionally, $1.56 \mathrm{~mL}$ of amprolium (Corid $9.6 \%$ oral solution; Merial Ltd., Duluth, GA) and $5.25 \mathrm{mg}$ of BioMos (mannanoligosaccharides; Alltech Inc., Brentwood, TN) per liter of milk was also added to each batch of pasteurized milk fed to calves during the study.

The study was a case-control design where calves affected with diarrhea were the cases and healthy neighbor housed calves with similar age $( \pm 2$ d) were chosen as controls. Diarrhea cases were defined as farm-diagnosed severe diarrhea, which were being treated with oral or intravenous fluids; cases were confirmed by a research team member after a complete physical examination of the affected calf was performed before fecal sample collection. Fecal samples from calves 2 to $8 \mathrm{~d}$ of age were collected by the research team (3 veterinarians) from calves with diarrheic (cases) and normal feces (controls). A total of 117 fecal samples were collected, of which 30 were from farm A, with 16 samples from control and 14 from diarrheic fecal samples; 11 were from farm B, with 4 samples from control and 7 from diarrheic fecal samples; and 76 samples were from farm C, with 41 samples from control and 35 from diarrheic fecal samples.

One of the limitations in the study design was the fact that GPA and NGPA groups did not have a neutral group present in any of the 3 farms belonging to the study. Ideally, for each individual calf in the antimicrobial-treated group, a corresponding control animal within the same farm should be present. However, the dairy farms that were not using GPA were unwilling to add such treatment to their management system and the farm using GPA was not willing to interrupt its use for some of the calves for the duration of the trial.

\section{Bacterial Isolation and Culture}

The fecal swabs were taken to the laboratory and cultured aerobically on MacConkey agar (Difco Labo- ratories Inc., Detroit, MI) at $37^{\circ} \mathrm{C}$, and E. coli colonies were distinguished by a purple-red color. Five typical E. coli colonies from the MacConkey agar cultures were picked and subsequently streaked on CHROMagar $E$. coli (CHROMagar, Paris, France) for isolation and further identification of $E$. coli strains. Selected colonies were stored in Luria-Bertani broth containing $20 \%$ glycerol at $-80^{\circ} \mathrm{C}$. Deoxyribonucleic acid was extracted using InstaGene matrix (Bio-Rad Laboratories, Hercules, CA).

\section{Antimicrobial Susceptibility Test}

Prior to the antimicrobial susceptibility test, all isolates were revived by subculture on Mueller-Hinton agar plates and incubated at $37^{\circ} \mathrm{C}$ for 16 to $18 \mathrm{~h}$ aerobically. For the antimicrobial susceptibility test, isolates were streaked onto Mueller-Hinton agar plates and incubated under aerobic conditions for 12 to $18 \mathrm{~h}$ at $37^{\circ} \mathrm{C}$. Isolates were suspended in $0.1 M$ sodium phosphate solution so that the turbidity was equivalent to that of a $0.5 \mathrm{McFarland}$ standard and streaked again onto 150-mm Mueller-Hinton agar plates. Antimicrobial susceptibility was determined by use of a Kirby-Bauer disk diffusion assay in accordance with guidelines published by the Clinical and Laboratory Standards Institute (CLSI; CLSI, 2008). Internal quality control was performed by inclusion of E. coli ATCC 25922. All susceptibility results of quality control strains complied with the quality control ranges. Antimicrobial susceptibility to 12 antibiotics for all isolates was assessed using the following panel: ampicillin $10 \mu \mathrm{g}$, amoxicillin-clavulanic acid $20 / 10 \mu \mathrm{g}$, cefepime $30 \mu \mathrm{g}$, ceftiofur $30 \mu \mathrm{g}$, ciprofloxacin $5 \mu \mathrm{g}$, enrofloxacin $5 \mu \mathrm{g}$, gentamicin $10 \mu \mathrm{g}$, nalidixic acid $30 \mu \mathrm{g}$, neomycin $30 \mu \mathrm{g}$, streptomycin $10 \mu \mathrm{g}$, tetracycline $30 \mu \mathrm{g}$, and trimethoprim-sulfamethoxazole $23.75 / 1.25 \mu \mathrm{g}$. The disks were applied to the plates, which were then incubated under aerobic conditions for 12 to $18 \mathrm{~h}$ at $37^{\circ} \mathrm{C}$, after which the diameters of the inhibition zones were measured and recorded.

Susceptibility of the isolates to most antimicrobial drugs was categorized (susceptible, intermediate, or resistant) by measuring the inhibition zone according to interpretive criteria adhering to the CLSI guidelines (CLSI, 2008). Because no ceftiofur and enrofloxacin inhibition zone measurements were available for bovine intestinal $E$. coli by CLSI guidelines, the breakpoints for gram-negative bovine respiratory agents were used as a guideline (CLSI, 2008). Since cefepime, ciprofloxacin, nalidixic acid, and streptomycin lacked a zone diameter interpretive standard from CSLI, an interpretation zone diameter from Enterobacteriaceae was used as reference (BD Diagnostics, 2007). No interpretive criterion was available for neomycin and the FDA-approved 
zone size recommendation was used as a guideline (BD Diagnostics, 2007).

\section{Detection of Virulence Genes}

One fecal $E$. coli isolate for each calf in the study was randomly selected for PCR amplification of known and putative virulence factors to identify the presence of any of 32 different virulence gene factors. These virulence factors were selected with the objective of screening specific virulence factors associated with disease and possible selection pressure for a specific virulence factor caused by antimicrobial resistance. The virulence gene factors selected for this study have been previously identified in calves with diarrhea and also related to extraintestinal pathogenic $E$. coli, creating a wide range for virulence factor screening, ideal for the proposed objective (Wenz et al., 2006; Gow and Waldner, 2009; Badouei et al., 2010; Bicalho et al., 2010).

All PCR reactions were performed in a $25-\mu \mathrm{L}$ volume using $24 \mu \mathrm{L}$ of $1 \mathrm{X}$ Green GoTaq Master Mix [made from 2X Green GoTaq Master Mix, consisting of Green GoTaq Reaction Buffer, $400 \mu M$ deoxyadenosine triphosphate (dATP), $400 \mu M$ deoxyguanosine triphosphate (dGTP), $400 \mu M$ deoxycytidine triphosphate (dCTP), $400 \mu M \quad 2^{\prime}$-deoxythymidine-5'-triphosphate (dTTP), and $3 \mathrm{mM} \mathrm{MgCl}$; Promega Corp., Madison, WI], 10 pmol of primer, and $1 \mu \mathrm{L}$ of DNA. All thermal cycling protocols were performed in a 2720 Thermal Cycler (Applied Biosystems, Foster City, CA). Negative controls consisting of the PCR mixture without DNA were included in all PCR runs. Amplification products were separated by electrophoresis through a $1.2 \%$ (wt/vol) agarose gel, stained with $0.5 \mu \mathrm{g}$ of ethidium bromide/mL, and visualized with a KODAK Gel Logic 100 Imaging System (GL 100; Eastman Kodak Co., Rochester, NY). Amplicons that had the expected molecular size were considered to be positives.

For further confirmation that the isolates belonged to the E. coli species, the 340-bp fragment of the bacterial $16 \mathrm{~S}$ rRNA-encoding gene was amplified by using the primers $16 \mathrm{SF}\left(5^{\prime}-\mathrm{GTT}\right.$ AAT ACC TTT GCT CAT TGA- $\left.3^{\prime}\right)$ and 16SR $\left(5^{\prime}-\right.$ ACC AGG GTA TCT AAT CCT GTT- $3^{\prime}$ ) as described by Malinen et al. (2003). All cultures that did not yield the 340-bp fragment in gel electrophoresis were excluded from the study. The genetic diversity of $E$. coli isolates was evaluated by randomly amplified polymorphic DNA (RAPD)-PCR with the informative primer $1283\left(5^{\prime}-\right.$ GCG ATC CCC A $-3^{\prime}$ ) as described previously (Wang et al., 1993). The images were visually analyzed; isolates from the same calf ( 5 isolates per calf) were compared and E. coli colonies with distinct genomic profiles were selected for subsequent analyses. After RAPD-PCR, 251 E. coli iso- lates, an average of 2.1 per positive calf, were selected for further analyses.

A multiplex $c d t$ PCR using 4 primer pairs was performed to detect $E$. coli isolates having sequences common to $c d t B$ genes, as described by Tóth et al. (2003). The thermal cycling conditions were $94^{\circ} \mathrm{C}$ for $5 \mathrm{~min}, 30$ cycles at $94^{\circ} \mathrm{C}$ for $1 \mathrm{~min}, 55^{\circ} \mathrm{C}$ for $1 \mathrm{~min}, 72^{\circ} \mathrm{C}$ for $1 \mathrm{~min}$, and a final step of $72^{\circ} \mathrm{C}$ for 10 min (Tóth et al., 2003). Escherichia coli isolates that yielded a PCR product size of $466 \mathrm{bp}$ were considered positive for the presence of $c d t B$ genes.

A multiplex 16plex PCR was conducted to assess $E$. coli isolates that had genes related to diarrheagenic $E$. coli; 16 pairs of primers were used in this PCR to detect 16 virulence factor (VF) genes (eaeA, esc V, ent, bfpB, EHEC-hly, stx1, stx2, ipaH, invE, astA, aggR, pic, elt, estIa, estIb, and uidA) as described (Antikainen et al., 2009). Cycling parameters were $98^{\circ} \mathrm{C}$ for $30 \mathrm{~s}, 30$ cycles at $98^{\circ} \mathrm{C}$ for $10 \mathrm{~s}, 63^{\circ} \mathrm{C}$ for $20 \mathrm{~s}, 72^{\circ} \mathrm{C}$ for $30 \mathrm{~s}$, and a final step at $72^{\circ} \mathrm{C}$ for 10 min (Antikainen et al., 2009). Furthermore, a multiplex 15plex PCR was performed to identify VF associated with uropathogenic E. coli (UPEC); 15 pairs of primers were used to detect $15 \mathrm{VF}$ genes [papAH, PapG (allele I), PapG (allele II), PapG (allele III), fimH, afa/draBC, sfa/focDE, hlyA, cnf1, iutA, fyuA, kpsMII, traT, ibeA, malX, PAI] as described by Moreno et al. (2005). To facilitate interpretation of the gel images, the primers from the $15 \mathrm{VF}$ gene groups were divided into 2 pools. Pool A was composed of 8 pairs of primers (Allele III f, Allele III r, Afa f, Afa r, sfa 1, sfa 2, hlyA f, hlyA r, cnf1, cnf2, FyuA f, FyuA r, TraT f, TraT r, Ibe10 f, and Ibe10 r), and pool B was composed of 7 pairs of primers (PapA f, PapA r, Allele I f, Allele I r, Allele II f, Allele II r, FimH f, FimH r, AerJ f, AerJ r, kps II f, kps II r, RPAi f, and RPAi r). The PCR of pools A and B were conducted separately; the thermal cycling protocol was $95^{\circ} \mathrm{C}$ for $12 \mathrm{~min}$, followed by 25 cycles at $94^{\circ} \mathrm{C}$ for $30 \mathrm{~s}, 63^{\circ} \mathrm{C}$ for $30 \mathrm{~s}$, and $68^{\circ} \mathrm{C}$ for $3 \mathrm{~min}$, followed by a final extension at $72^{\circ} \mathrm{C}$ for $10 \mathrm{~min}$ (Moreno et al., 2005).

\section{DNA Gyrase Amplification, Sequencing, and Phylogenetic Analysis}

For this molecular analysis, 31 randomly selected isolates, 16 from diarrheic calves and 15 from control calves (non-diarrheic), from NGPA farms were used. Chromosomal DNA was extracted with InstaGene matrix (Bio-Rad Laboratories) and amplified by PCR in a 2720 Thermal Cycler (Applied Biosystems). The PCR conditions and primers used were as described previously (Fukushima et al., 2002). The purified PCR products were sequenced using DNA Illumina PairedEnd sequencing at the Cornell University Life Sciences 
Core Laboratory Center (CLC, Ithaca, NY). The PCR fragments were determined by using the sequencing primers UP1S (5'-AAGTCATCATGACCGTTCTGCA-3') and UP2Sr (5'-AGCAGGGTACGGATGTGCGAGCC-3'; Fukushima et al., 2002). For phylogenetic analysis, DNA gyrase B (gyrB) sequences from 31 fecal E. coli genetically distinct bacteria were aligned with 1 Escherichia fergusonii strain and 32 strains of E. coli with known pathotypes.

The gyr B sequences were aligned using the ClustalW algorithm using the software Geneious 4.8.4 (Biomatters Ltd., Auckland, New Zealand) and adjusting for 1,050 bases. All bacterial strains used in this phylogenetic analysis, with the exception of the fecal E. coli, have had their whole chromosomal genomes sequenced and were publically available at the National Center for Biotechnology Information (NCBI) website at the time the analysis was performed (NCBI, 2011).

Detailed information regarding $E$. coli pathotype and strain identification is included in the phylogenetic tree. Evolutionary relationships were inferred by using the neighbor-joining method, and the evolutionary distances were computed by using the Tamura-Nei method (Tamura and Nei, 1993). Bootstrap values were calculated from 1,000 replicate analyses.

\section{Statistical Analysis}

To analyze the effect of GPA on the inhibition zone diameter, a general linear model was fitted to the data using the MIXED procedure of SAS (SAS Inst. Inc., Cary, NC). The outcome variable was antibiotic-specific inhibition zone diameter $(\mathrm{mm})$, which was modeled as a Gaussian (normally distributed data) variable. The assumption that the residuals were normally distributed was satisfied by visually evaluating the distribution plot of the studentized residuals. The following variables were offered to the model: GPA status of the farm (GPA and NGPA) and health status of the calf (diarrheic and non-diarrheic).

Bacterial isolates were categorized as susceptible, intermediate, or resistant in accordance with interpretive methods and breakpoints described previously. Several ordinal logistic regression models, one for each tested antibiotic, were fitted to the data using the ologit procedure of STATA (STATA 9.2; StataCorp LP, College Station, TX). The ordinal variable antibiotic susceptibility (susceptible, intermediate, or resistant) was used as the dependent variable and the variables GPA status of the farm (GPA and NGPA) and health status of the calf (diarrheic and non-diarrheic) were offered to the model as independent variables. To evaluate if the fitted models complied with the proportional odds assumption of ordinal logistic regression, the Brant test was performed using the brant command in STATA; all fitted models complied with the assumption.

Chi-squared tests were performed in Jump 7.0 (SAS Institute Inc.) to assess the difference in the presence of genes encoding virulence factors between the isolates recovered from Holstein calves in the GPA and NGPA groups, and with diarrheic versus non-diarrheic calves. For all statistical models and tests, variables were considered significant when a $P<0.05$ was observed.

\section{RESULTS}

\section{Antimicrobial Resistance in E. coli}

All isolates were susceptible to ciprofloxacin, an antibiotic widely used in human medicine that is similar to enrofloxacin from the fluoquinolone class, and cefepime, a fourth-generation cephalosporin used in human medicine that has greater activity against both gram-negative and gram-positive organisms than thirdgeneration cephalosporin agents. However, $87 \%$ of the isolates were resistant to tetracycline $(\mathrm{n}=251)$, and about $70 \%$ of the isolates were resistant to ampicillin, streptomycin and sulfamethoxazole-trimethoprim. For amoxicillin-clavulanic acid and ceftiofur, more than $30 \%$ of isolates were intermediary (Figure 1). Significantly smaller diameter zones for the disk diffusion test were observed in the GPA group for tetracycline, ampicillin, streptomycin, sulfamethoxazole-trimethoprim, amoxicillin-clavulanic acid, ceftiofur, and cefepime when compared with the NGPA group (Table 1). Isolates from non-diarrheic and diarrheic fecal samples did not indicate differences for the diameter zones of the disk diffusion test (Table 1).

Of the 251 isolates, $84 \%(\mathrm{n}=211)$ were resistant to at least 2 antimicrobial agents tested, and multidrug resistance ( $\geq 3$ antimicrobial agents) was observed in $81 \%$ (n $=204) E$. coli isolates. From the 43 multidrug resistance profiles found, the most frequent profile was for tetracycline-ampicillin-streptomycin-sulfamethoxazole-trimethoprim (Te-Am-S-Sxt), observed in $25 \%$ of isolates (n $=62$; Table 2). The second most prevalent multidrug resistance profile was for tetracycline-ampicillin-streptomycin-amoxicillin-clavulanic acid (Te-Am-S-Amc), observed in 42 isolates with a $17 \%$ incidence (Table 2).

The odds of antimicrobial resistance occurring was compared between the NGPA and GPA groups. The NGPA group had statistically significantly lower odds $(P>0.005)$ for antimicrobial resistance for most of the antimicrobials tested (Table 3 ).

\section{Presence of Known and Putative Virulence Factor Genes}

Of the 32 virulence factor genes screened, 21 were identified in at least 1 of the 117 isolates. Virulence fac- 
Table 1. Least squares means of inhibition zone diameters ( $\mathrm{mm} ; 95 \% \mathrm{CI}$ in parentheses) and respective $P$-values by milk treatment group [no growth-promoting antimicrobials added to milk (NGPA) and growth-promoting antimicrobials added to milk (GPA)] and health status group (diarrheic and non-diarrheic) for Escherichia coli isolates from fecal samples of dairy calves 2 to 8 d of age $(\mathrm{n}=251$ isolates)

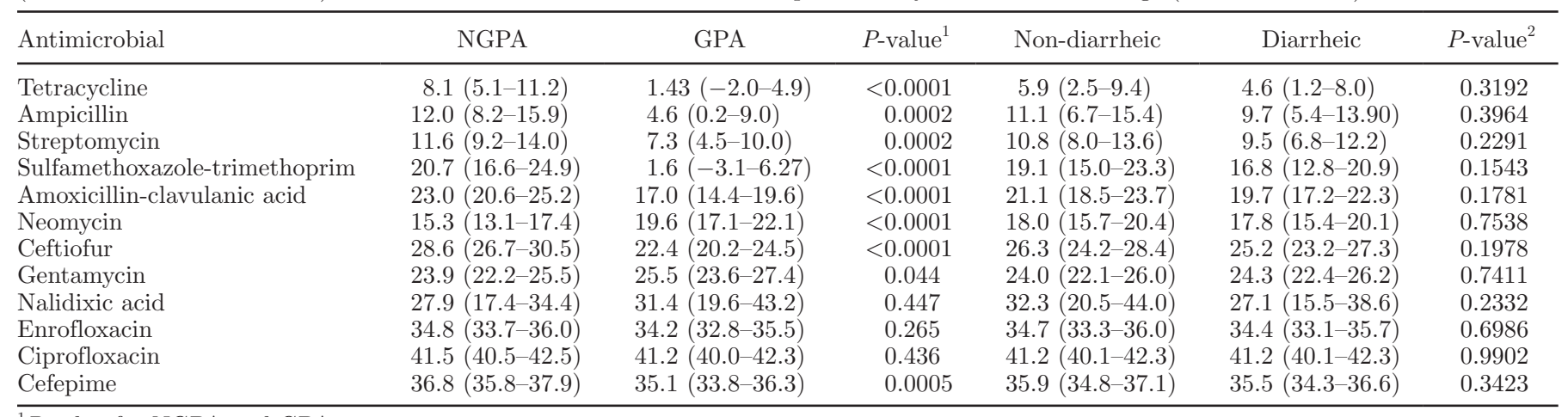

${ }^{1} P$-value for NGPA and GPA.

${ }^{2} P$-value for calves presenting or not presenting diarrhea.

tor fyuA had a higher prevalence in the NGPA group, and allele II from the pap $G$ gene had a higher prevalence in the GPA group. When comparing the control and diarrheic groups, the only virulence factors that had a statistically significant value were stx1, which had a higher prevalence in the control group, and hly A, which had a higher prevalence in the diarrheic group (Table 4).

\section{DNA Gyrase Phylogenetic Analysis}

For phylogenetic analysis, DNA gyrase B (gyr B) sequences from 31 fecal $E$. coli genetically distinct bacteria were aligned with 1 Escherichia fergusonii strain and 32 strains of E. coli with known pathotypes. Figure
2 shows the phylogenetic tree for these species based on the gyrB gene sequence. As expected, the majority $(\mathrm{n}=10)$ of the control isolates collected from calves that did not present diarrhea were grouped adjacent to the nonpathogenic E. coli. For the isolates collected from calves presenting diarrhea, 38\% $(\mathrm{n}=6)$ were grouped in a clade together with enterohemorrhagic $E$. coli $($ EHEC) and $25 \%(\mathrm{n}=4)$ were grouped in a clade adjacent to enterotoxigenic E. coli (ETEC). A few (n = 4) control isolates also were grouped together with the EHEC clade.

\section{DISCUSSION}

The use of antimicrobials as a preventive measure against calf diarrhea has been proven to be beneficial in

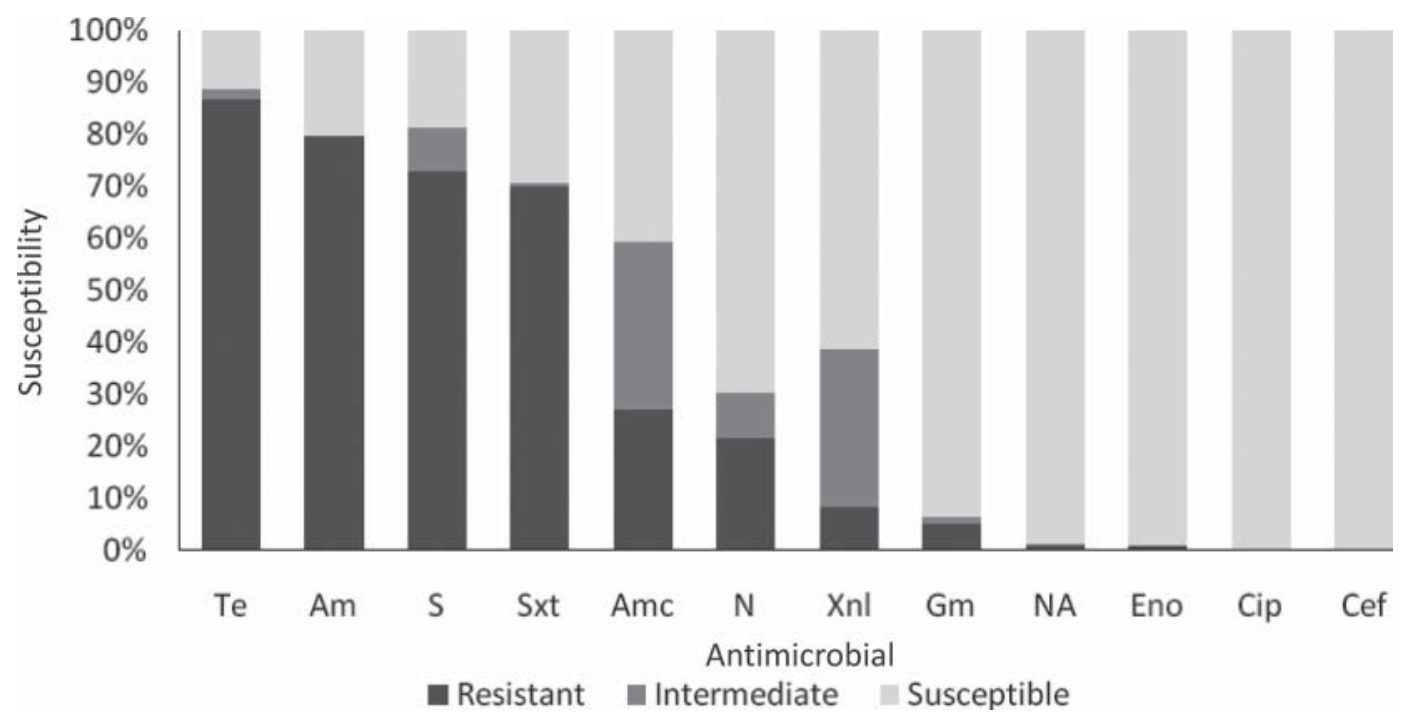

Figure 1. Antimicrobial susceptibility of Escherichia coli isolates from fecal samples of calves for the 12 antimicrobials tested (n $=251$ isolates). Te = tetracycline; Am = ampicillin; $\mathrm{S}=$ streptomycin; Sxt = sulfamethoxazole-trimethoprim; Amc = amoxicillin-clavulanic acid; $\mathrm{N}=$ neomycin; Xnl = ceftiofur; $\mathrm{Gm}=$ gentamycin; NA = nalidixic acid; Eno = enrofloxacin; $\mathrm{Cip}=$ ciprofloxacin; Cef = cefepime. 
Table 2. Antimicrobial multiresistance profiles for Escherichia coli isolates recovered from fecal samples of calves

\begin{tabular}{lcc}
\hline $\begin{array}{l}\text { Resistance } \\
\text { phenotype }\end{array}$ & Number & Percentage \\
\hline Te-Am-S-Amc-N & 4 & 1.59 \\
Te-Am-Sxt-N & 4 & 1.59 \\
Te-Am-S-Sxt-Xnl & 4 & 1.59 \\
Te-Am-S-Sxt-Amc-N & 5 & 1.99 \\
Te-Am-S-N & 7 & 2.79 \\
Te-Am-S & 8 & 3.19 \\
Te-Am-S-Sxt-Amc-Xnl & 9 & 3.59 \\
Te-Am-Sxt & 11 & 4.38 \\
Te-Am-S-Sxt-N & 19 & 7.57 \\
Te-Am-S-Amc & 42 & 16.73 \\
Te-Am-S-Sxt & 62 & 24.70 \\
Other & 76 & 30.28 \\
\hline
\end{tabular}

${ }^{1} \mathrm{Te}=$ tetracycline; $\mathrm{Am}=$ ampicillin; $\mathrm{S}=$ streptomycin; Sxt $=$ sulfamethoxazole-trimethoprim; Amc $=$ amoxicillin-clavulanic acid; $\mathrm{N}=$ neomycin; $\mathrm{Xnl}=$ ceftiofur.

${ }^{2}$ Antimicrobial multiresistant profiles with less than 4 strains.

decreasing morbidity and mortality (Berge et al., 2009). However, this practice has also led to the emergence of antimicrobial-resistant E. coli in cattle, capable of colonizing humans and causing infections with limited therapeutic options (Hammerum and Heuer, 2009). In the present study, the use of antimicrobials in the GPA group showed that for 8 of the 12 antimicrobials tested, a smaller diameter of inhibition zone was observed when compared with farms in the NGPA group.
In veterinary medicine, $\beta$-lactams and tetracyclines are extensively used. Our findings show that at least $97 \%$ of isolates were resistant to all antimicrobial agents from these classes in the GPA group, versus $74 \%$ in the NGPA group. Bacterial resistance to $\beta$-lactam can be achieved by 3 mechanisms: the production of $\beta$-lactam-hydrolyzing $\beta$-lactamase enzymes, the utilization of $\beta$-lactam-insensitive cell wall transpeptidases, and the active expulsion of $\beta$-lactam molecules from gram-negative cells by way of efflux pumps (Wilke et al., 2005).

Tetracycline resistance originates in most cases from acquiring resistance genes (tet genes) and not from mutation (Chopra and Roberts, 2001). The 2 most relevant resistance mechanisms associated with tet genes are efflux pumping and ribosomal protection (von Baum and Marre, 2005). Resistance to tetracycline was 12.4 times higher for the GPA group when compared with the NGPA group.

The odds of ceftiofur resistance occurring in the GPA group was 4.25 when compared with the NGPA group. Extended-spectrum $\beta$-lactamase (ESBL) E. coli consist of $\beta$-lactamases capable of conferring bacterial resistance to penicillins, first-, second-, and third-generation cephalosporins, and aztreonam by hydrolysis of theses antibiotics (Paterson and Bonomo, 2005; Lee et al., 2010). Farm animals, as reservoirs of ESBL E. coli, have been studied (Briñas et al., 2003; Liebana et al.,

Table 3. Outcome of logistic regression evaluating the effect of antimicrobial resistance of Escherichia coli isolates for fecal samples from calves in a group with no growth-promoting antimicrobials added to milk (NGPA) and in a group with growth-promoting antimicrobials added to milk (GPA)

\begin{tabular}{|c|c|c|c|c|c|}
\hline Antimicrobial & Status & NGPA & GPA & OR & $P$-value ${ }^{1}$ \\
\hline \multirow[t]{3}{*}{ Sulfamethoxazole-trimethoprim } & Susceptible & 68.7 & 5.1 & Reference & \multirow{3}{*}{$>0.005$} \\
\hline & Intermediate & 0.0 & 0.6 & & \\
\hline & Resistant & 31.2 & 94.2 & 36.1 & \\
\hline \multirow[t]{3}{*}{ Tetracycline } & Susceptible & 25 & 2.6 & Reference & \multirow{3}{*}{$>0.005$} \\
\hline & Intermediate & 4.12 & 0.6 & & \\
\hline & Resistant & 70.8 & 96.8 & 12.4 & \\
\hline \multirow{3}{*}{ Ampicillin } & Susceptible & 40.63 & 7.7 & Reference & \multirow{3}{*}{$>0.005$} \\
\hline & Intermediate & 0.0 & 0.0 & & \\
\hline & Resistant & 59.4 & 92.3 & 8.1 & \\
\hline \multirow[t]{3}{*}{ Amoxicillin-clavulanic acid } & Susceptible & 68.7 & 23.2 & Reference & \multirow{3}{*}{$>0.005$} \\
\hline & Intermediate & 18.7 & 40.65 & 62 & \\
\hline & Resistant & 12.5 & 36.13 & 0.2 & \\
\hline \multirow[t]{3}{*}{ Streptomycin } & Susceptible & 39.6 & 5.8 & Reference & \multirow{3}{*}{$>0.005$} \\
\hline & Intermediate & 7.3 & 9.0 & & \\
\hline & Resistant & 53.1 & 85.2 & 0.80 & \\
\hline \multirow[t]{3}{*}{ Ceftiofur } & Susceptible & 81.2 & 49 & Reference & \multirow{3}{*}{$>0.005$} \\
\hline & Intermediate & 13.5 & 40.6 & & \\
\hline & Resistant & 5.2 & 10.3 & $4_{4}$ & \\
\hline \multirow[t]{3}{*}{ Gentamycin } & Susceptible & 90.6 & 95.5 & Reference & \multirow{3}{*}{0.14} \\
\hline & Intermediate & 2.1 & 0.6 & & \\
\hline & Resistant & 7.3 & 3.9 & 0.46 & \\
\hline \multirow[t]{3}{*}{ Neomycin } & Susceptible & 50 & 81.9 & Reference & \multirow{3}{*}{$>0.005$} \\
\hline & Intermediate & 8.3 & 9.0 & 019 & \\
\hline & Resistant & 41.7 & 9.0 & 0.19 & \\
\hline
\end{tabular}

${ }^{1}$ Cefepime, ciprofloxacin, nalidixic acid, and enrofloxacin were not included in the analysis because no strains showed any degree of resistance to these antibiotics. 
Table 4. Prevalence of virulence factor genes of Escherichia coli and respective $P$-values between control or diarrheic groups and farms that used growth-promoting antibiotic (GPA) or did not use growth-promoting antibiotic (NGPA) in the milk fed to the calves ${ }^{1}$

\begin{tabular}{lcccccc}
\hline $\begin{array}{l}\text { Virulence } \\
\text { factor }\end{array}$ & $\begin{array}{c}\text { Control } \\
(\%)\end{array}$ & $\begin{array}{c}\text { Diarrheic } \\
(\%)\end{array}$ & $\begin{array}{c}P \text {-value } \\
\text { fimH }\end{array}$ & $\begin{array}{c}\text { GPA } \\
(\%)\end{array}$ & $\begin{array}{c}\text { NGPA } \\
(\%)\end{array}$ & $P$-value \\
\hline traT & 100 & 98.21 & 0.22 & 100 & 97.56 & 0.14 \\
uidA & 96.72 & 94.64 & 0.57 & 97.37 & 92.68 & 0.24 \\
iutA & 91.8 & 96.43 & 0.28 & 93.42 & 95.12 & 0.7 \\
ibeA & 86.89 & 83.93 & 0.65 & 81.58 & 92.68 & 0.08 \\
fyuA & 70.49 & 73.21 & 0.74 & 73.68 & 68.29 & 0.53 \\
PapG (allele II) & 52.46 & 50 & 0.79 & 38.16 & 75.61 & $<0.0001$ \\
papAH & 40.98 & 32.14 & 0.32 & 46.05 & 19.51 & 0.003 \\
astA & 34.43 & 26.79 & 0.37 & 30.26 & 31.71 & 0.87 \\
escV & 32.79 & 37.5 & 0.59 & 39.47 & 26.83 & 0.16 \\
cdt & 27.87 & 23.21 & 0.56 & 28.95 & 19.51 & 0.25 \\
malX,PAI & 13.11 & 10.71 & 0.68 & 11.84 & 12.2 & 0.95 \\
stx1 & 8.2 & 5.36 & 0.54 & 5.26 & 9.76 & 0.36 \\
sfa/focDE & 6.56 & 0 & 0.02 & 3.95 & 2.44 & 0.66 \\
Afa/draBC & 4.92 & 7.14 & 0.61 & 5.26 & 7.32 & 0.65 \\
PapG (allele I) & 1.64 & 5.36 & 0.26 & 2.63 & 4.88 & 0.53 \\
PapG (allele III) & 1.64 & 1.79 & 0.95 & 2.63 & 0 & 0.18 \\
kpsMII & 1.64 & 0 & 0.25 & 0 & 2.44 & 0.14 \\
stx2 & 1.64 & 5.36 & 0.26 & 3.95 & 2.44 & 0.66 \\
EHEC-hly & 0 & 1.79 & 0.22 & 0 & 2.44 & 0.14 \\
hlyA & 0 & 1.79 & 0.22 & 0 & 2.44 & 0.14 \\
IA total & 0 & 5.36 & 0.03 & 1.32 & 4.88 & 0.25
\end{tabular}

${ }^{1} \mathrm{~A}$ total of 117 isolates, 61 from diarrheic and 56 from non-diarrheic calves, and 76 from the GPA group and 41 from the NPGA group were tested.

${ }^{2} P$-value for calves presenting or not presenting diarrhea.

${ }^{3} P$-value for NGPA and GPA. Note: only virulence factors identified in at least 1 of the strains tested are shown.

2006: Carattoli, 2008), but still additional data are necessary for evaluating the epidemiological effects of supplementing antimicrobials to calves on the incidence of this worldwide concern (Bush, 2008; Cantón et al., 2008; Hawkey, 2008; Villegas et al., 2008).

In the present study, the GPA group had 36.7-fold higher odds for resistance to sulfamethoxazole-trimethoprim when compared with the NGPA group. The hypothesis for this higher level of resistance is that the use of sodium sulfamethazine, a drug belonging to the sulfonamide class, may have caused antimicrobial resistance to sulfamethoxazole-trimethoprim by coselection. Rosengren et al. (2007) reported a direct relationship between the use of sulfonamides (other than sulfamethoxazole-trimethoprim) on resistance to sulfamethoxazole-trimethoprim in piglets. The study showed that piglets had 4.6 higher odds of resistant strains if receiving sulfonamides in the food and water. However, the measured phenotype could have been too crude of a metric to adequately explain co-selection because antimicrobial exposure did not appear to coselect for all of the genes encoding a given phenotype (Rosengren et al., 2007, 2009).

The most prevalent multidrug resistant pattern within all isolates in the present study was tetracycline-ampicillin- streptomycin-sulfamethoxazole-trimethoprim
(Te-Am-S-Sxt), with $25 \%$ of isolates resistant. Similar resistance patterns to these antibiotics have been found for fecal E. coli from cattle (Carson et al., 2008; Lee et al., 2010), with $>59 \%$ of isolates having a resistance pattern to tetracycline, streptomycin, and sulfadiazine (Te-S-Sxt; Khachatryan et al., 2006).

It is an accepted dogma that an organism pays a physiological price for antimicrobial resistance, particularly where resistance is mediated by chromosomal mutations. This biological cost is commonly referred as fitness cost (Pope et al., 2010). Most chromosomal mutations that cause antibiotic resistance impose a fitness cost on bacteria, which may include decreased virulence and lower growth rates (Maisnier-Patin et al., 2002). In the present study, the virulence factor fyuA had a higher prevalence in the NGPA group, and allele II from the $\operatorname{pap} G$ gene had a higher prevalence in the GPA group. When comparing the control and diarrheic groups, the only virulence factors that had a statistically significant value were stx1, which had a higher prevalence in the control group, and hlyA, which had a higher prevalence in the diarrheic group.

The absence of an association between phenotypic antimicrobial resistance and virulence gene factors in the GPA group may be explained by fitness cost of antimicrobial resistance genes (Gow and Waldner, 


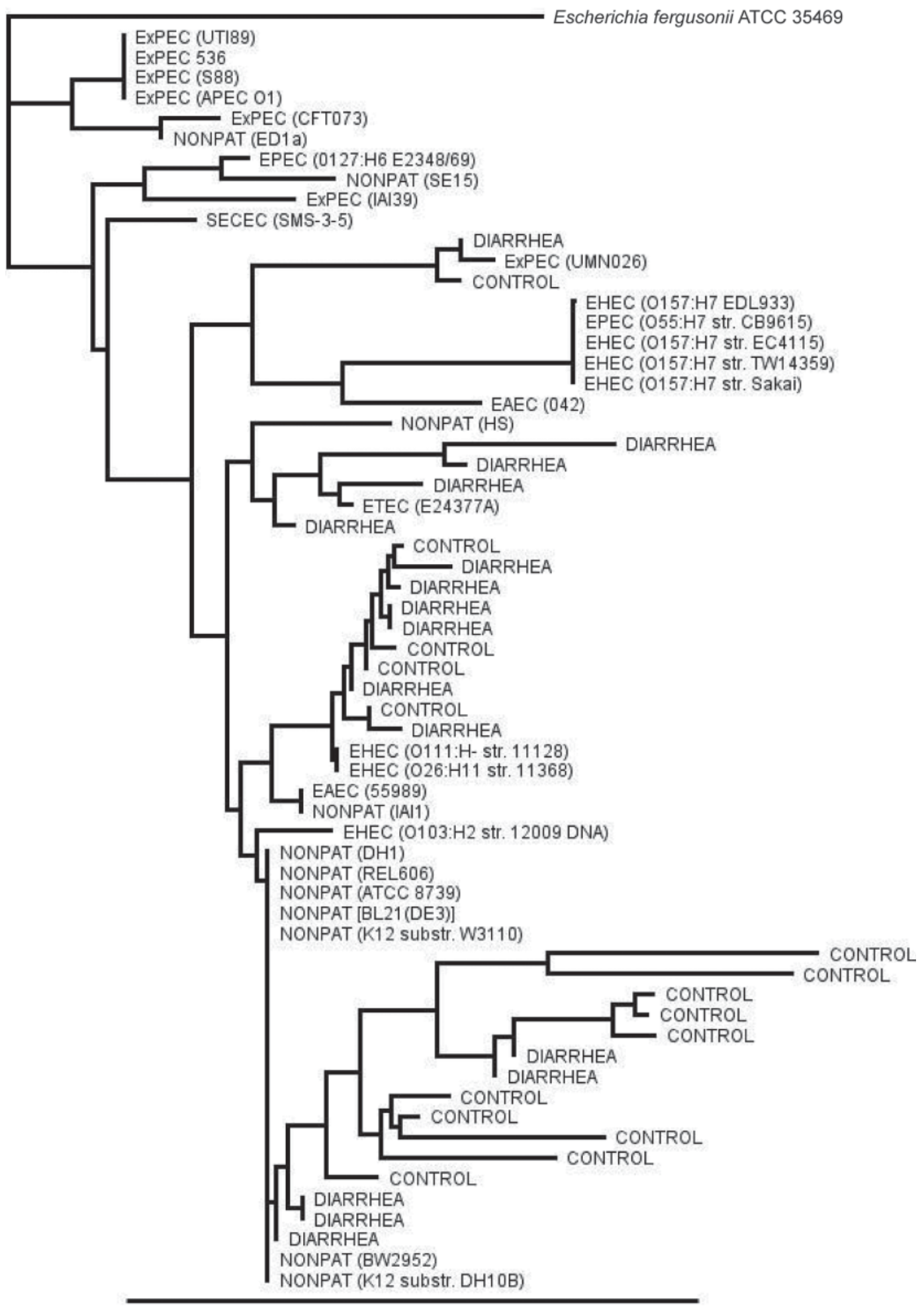

0.02

Figure 2. Phylogenetic tree based on DNA gyrase B ( gyrB) sequences illustrating the evolutionary distance between 31 genetically distinct strains of fecal Escherichia coli from Holstein calves and 32 strains of E. coli with known pathotypes. ExPEC = extraintestinal E. coli; EAEC $=$ enteroaggregative $E$. coli EHEC = enterohemorrhagic E. coli; NONPAT = nonpathogenic E. coli; SECEC = E. coli strain SMS-3-5; EPEC $=$ enteropathogenic E. coli; ETEC = enterotoxigenic E. coli. 
2009; Rosengren et al., 2009). However, the fitness cost from antimicrobials did not extend to a point where isolates in the GPA group presented a significantly lower incidence of virulence factor when compared with the NPGA group; this could have occurred from the development by $E$. coli of a compensatory evolution mechanism to bypass a higher fitness cost associated with carrying antimicrobial resistance genes (Andersson and Hughes, 2010). More research would be necessary to identify specify compensatory mechanisms to confirm this hypothesis for E. coli.

The lack of a higher incidence of virulence factors in the diarrheic group was an unexpected surprise in the study. Within hypotheses raised from such findings, one includes the fact that even though $E$. coli is the most common agent causing diarrhea in calves during the first week of life, pathogenic agents other than $E$. coli could have been responsible for the diarrhea in the calves used for fecal sampling, consequently removing an expected higher virulence of E. coli isolates. Another possibility would be in agreement with the findings of Schierack et al. (2006), where virulence factor-carrying $E$. coli strains in pigs were a normal part of intestinal bacterial populations and high numbers of $E$. coli harboring virulence genes did not necessarily correlate with disease (Schierack et al., 2006). The identification of virulence factors does not necessary imply the necessity of presence of clinical disease and previous research has shown healthy cattle harboring $E$. coli with various virulence gene factors (China et al., 1998; Orden et al., 2002).

Finally, DNA gyrase sequences from 31 genetically distinct fecal $E$. coli from diarrheic and control calves belonging to the NGPA group were aligned with a variety of enteric bacteria. The phylogenetic tree (Figure 2) revealed that most of the isolates tested clustered into 3 major clades: the first clade, where $25 \%$ of the isolates from diarrheic calves were located together with the reference enterotoxigenic E. coli strain (E24377A); the second clade, where $38 \%$ of the isolates from diarrheic calves were located together with the reference strains EHEC (O111:H-str. 11128), EHEC (O26:H11 str. 11368), nonpathogenic E. coli strain (A-1), and enteroaggregative $E$. coli (EAEC) 55989; and the third clade where $67 \%$ of the control isolates were grouped together with several nonpathogenic reference $E$. coli strains. For the phylogenetic analysis of closely related bacterial strains, the use of the gyr $\mathrm{B}$ gene has been suggested to be preferred over the use of the 16S rRNA region because the gyr $\mathrm{B}$ gene evolves at a higher rate, providing more heterogeneity for the analysis (Fukushima et al., 2002). The authors suggest that the gyrB region could have high reliability for the identification of pathogenic bacteria.

\section{CONCLUSIONS}

We observed a higher occurrence of antimicrobial resistance to $E$. coli recovered from fecal samples of preweaned dairy calves that received GPA in the milk, highlighting the need for prudent use of antimicrobial agents in veterinary medicine to decrease the incidence of antimicrobial-resistant bacteria for both animals and humans. Of the 32 virulence factors screened, only 21 were detected in the study population and the incidence of only 1 virulence factor was statistically significant in each of the diarrheic status (diarrheic or non-diarrheic) and treatment status (NGPA or GPA) groups. Even though a higher incidence of virulence factors was expected for $E$. coli isolates from diarrheic calves, no statistical difference was observed for most of the virulence factors screened for in the study.

\section{REFERENCES}

Alexander, T. W., G. D. Inglis, L. J. Yanke, E. Topp, R. R. Read, T. Reuter, and T. A. McAllister. 2010. Farm-to-fork characterization of Escherichia coli associated with feedlot cattle with a known history of antimicrobial use. Int. J. Food Microbiol. 137:40-48.

Andersson, D. I., and D. Hughes. 2010. Antibiotic resistance and its cost: Is it possible to reverse resistance? Nat. Rev. Microbiol. $8: 260-271$

Antikainen, J., E. Tarkka, K. Haukka, A. Siitonen, M. Vaara, and J. Kirveskari. 2009. New 16-plex PCR method for rapid detection of diarrheagenic Escherichia coli directly from stool samples. Eur. J. Clin. Microbiol. Infect. Dis. 28:899-908.

Badouei, M. A., T. Z. Salehi, M. R. Khorasgani, H. Tadjbakhsh, G. N. Brujeni, and M. G. Nadalian. 2010. Virulence gene profiles and intimin subtypes of Shiga toxin-producing Escherichia coli isolated from healthy and diarrheic calves. Vet. Rec. 167:858-861.

BD Diagnostics. 2007. BD BBL Sensi-Disc antimicrobial susceptibility test discs. BD Diagnostics, Sparks, MD. March 20, 2011. http:// www.bd.com/ds/technicalCenter/misc/ch_8_2052.pdf.

Berge, A. C. B., P. Lindeque, D. A. Moore, and W. M. Sischo. 2005. A clinical trial evaluating prophylactic and therapeutic antibiotic use on health and performance of preweaned calves. J. Dairy Sci. $88: 2166-2177$.

Berge, A. C., D. A. Moore, T. E. Besser, and W. M. Sischo. 2009 Targeting therapy to minimize antimicrobial use in preweaned calves: Effects on health, growth, and treatment costs. J. Dairy Sci. 92:4707-4714.

Bicalho, R. C., V. S. Machado, M. L. Bicalho, R. O. Gilbert, A. G. Teixeira, L. S. Caixeta, and R. V. Pereira. 2010. Molecular and epidemiological characterization of bovine intrauterine Escherichia coli. J. Dairy Sci. 93:5818-5830.

Briñas, L., M. A. Moreno, T. Teshager, M. Zarazaga, Y. Sáenz, C. Porrero, L. Dominguez, and C. Torres. 2003. $\beta$-Lactamase characterization in Escherichia coli isolates with diminished susceptibility or resistance to extended-spectrum cephalosporins recovered from sick animals in Spain. Microb. Drug Resist. 9:201-209.

Bush, K. 2008. Extended-spectrum $\beta$-lactamases in North America, 1987-2006. Clin. Microbiol. Infect. 14(Suppl. 1):134-143.

Cantón, R., A. Novais, A. Valverde, E. Machado, L. Peixe, F. Baquero, and T. M. Coque. 2008. Prevalence and spread of extendedspectrum beta-lactamase-producing Enterobacteriaceae in Europe. Clin. Microbiol. Infect. 14(Suppl 1):144-153.

Carattoli, A. 2008. Animal reservoirs for extended spectrum $\beta$-lactamase producers. Clin. Microbiol. Infect. 14(Suppl. 1):117123.

Carson, C. A., R. Reid-Smith, R. J. Irwin, W. S. Martin, and S. A. McEwen. 2008. Antimicrobial resistance in generic fecal Esch- 
erichia coli from 29 beef farms in Ontario. Can. J. Vet. Res. 72:119-128.

China, B., V. Pirson, and J. Mainil. 1998. Prevalence and molecular typing of attaching and effacing Escherichia coli among calf populations in Belgium. Vet. Microbiol. 63:249-259.

Chopra, I., and M. Roberts. 2001. Tetracycline antibiotics: Mode of action, applications, molecular biology, and epidemiology of bacterial resistance. Microbiol. Mol. Biol. Rev. 65:232-260

CLSI (Clinical and Laboratory Standards Institute). 2008. Performance standards for antimicrobial disk and dilution susceptibility tests for bacteria collected from animals; Approved standard. 3rd ed. Vol. 28 No. 8. Clinical and Laboratory Standards Institute, Wayne, PA.

Fukushima, M., K. Kakinuma, and R. Kawaguchi. 2002. Phylogenetic analysis of Salmonella, Shigella, and Escherichia coli strains on the basis of the gyrB gene sequence. J. Clin. Microbiol. 40:2779-2785.

Gow, S. P., and C. L. Waldner. 2009. Antimicrobial resistance and virulence factors stx1, stx2, and eae in generic Escherichia coli isolates from calves in Western Canadian cow-calf herds. Microb. Drug Resist. 15:61-67.

Hammerum, A. M., and O. E. Heuer. 2009. Human health hazards from antimicrobial-resistant Escherichia coli of animal origin. Clin. Infect. Dis. 48:916-921.

Hawkey, P. M. 2008. Prevalence and clonality of extended-spectrum $\beta$-lactamases in Asia. Clin. Microbiol. Infect. 14(Suppl 1):159 165 .

Khachatryan, A. R., T. E. Besser, D. D. Hancock, and D. R. Call. 2006. Use of a nonmedicated dietary supplement correlates with increased prevalence of streptomycin-sulfa-tetracycline-resistant Escherichia coli on a dairy farm. Appl. Environ. Microbiol. 72:4583-4588.

Lee, J. H., I. K. Bae, and S. Hee Lee. 2010. New definitions of extended-spectrum $\beta$-lactamase conferring worldwide emerging antibiotic resistance. Med. Res. Rev. doi:10.1002/med.20210.

Liebana, E., M. Batchelor, K. L. Hopkins, F. A. Clifton-Hadley, C. J. Teale, A. Foster, L. Barker, E. J. Threlfall, and R. H. Davies. 2006. Longitudinal farm study of extended-spectrum $\beta$-lactamasemediated resistance. J. Clin. Microbiol. 44:1630-1634.

Maisnier-Patin, S., O. G. Berg, L. Liljas, and D. I. Andersson. 2002. Compensatory adaptation to the deleterious effect of antibiotic resistance in Salmonella typhimurium. Mol. Microbiol. 46:355-366.

Malinen, E., A. Kassinen, T. Rinttilä, and A. Palva. 2003. Comparison of real-time PCR with SYBR green I or 5'-nuclease assays and dot-blot hybridization with rDNA-targeted oligonucleotide probes in quantification of selected faecal bacteria. Microbiology 149:269-277.

Moreno, E., I. Planells, G. Prats, A. M. Planes, G. Moreno, and A. Andreu. 2005. Comparative study of Escherichia coli virulence determinants in strains causing urinary tract bacteremia versus strains causing pyelonephritis and other sources of bacteremia. Diagn. Microbiol. Infect. Dis. 53:93-99.

NCBI (National Center for Biotechnology Information). 2011. National Center for Biotechnology Information. Accessed Jan. 7, 2011. http://www.ncbi.nlm.nih.gov.
Orden, J. A., D. Cid, J. A. Ruiz-Santa-Quiteria, S. Garcia, S. Martinez, and R. de la Fuente. 2002. Verotoxin-producing Escherichia coli (VTEC), enteropathogenic Escherichia coli (EPEC) and necrotoxigenic Escherichia coli (NTEC) isolated from healthy cattle in Spain. J. Appl. Microbiol. 93:29-35.

Paterson, D. L., and R. A. Bonomo. 2005. Extended-spectrum $\beta$-lactamases: A clinical update. Clin. Microbiol. Rev. 18:657-686.

Pope, C. F., T. D. McHugh, and S. H. Gillespie. 2010. Methods to determine fitness in bacteria. Methods Mol. Biol. 642:113-121.

Rosengren, L. B., C. L. Waldner, and R. J. Reid-Smith. 2009. Associations between antimicrobial resistance phenotypes, antimicrobial resistance genes, and virulence genes of fecal Escherichia coli isolates from healthy grow-finish pigs. Appl. Environ. Microbiol. 75:1373-1380

Rosengren, L. B., C. L. Waldner, R. J. Reid-Smith, P. M. Dowling, and J. C. Harding. 2007. Associations between feed and water antimicrobial use in farrow-to-finish swine herds and antimicrobial resistance of fecal Escherichia coli from grow-finish pigs. Microb. Drug Resist. 13:261-269.

Schierack, P., H. Steinrück, S. Kleta, and W. Vahjen. 2006. Virulence factor gene profiles of Escherichia coli isolates from clinically healthy pigs. Appl. Environ. Microbiol. 72:6680-6686.

Tamura, K., and M. Nei. 1993. Estimation of the number of nucleotide substitutions in the control region of mitochondrial DNA in humans and chimpanzees. Mol. Biol. Evol. 10:512-526.

Tóth, I., F. Hérault, L. Beutin, and E. Oswald. 2003. Production of cytolethal distending toxins by pathogenic Escherichia coli strains isolated from human and animal sources: Establishment of the existence of a new $c d t$ variant (type IV). J. Clin. Microbiol. 41:4285-4291.

van den Bogaard, A. E., and E. E. Stobberingh. 2000. Epidemiology of resistance to antibiotics. Links between animals and humans. Int. J. Antimicrob. Agents 14:327-335.

Villegas, M. V., J. N. Kattan, M. G. Quinteros, and J. M. Casellas. 2008. Prevalence of extended-spectrum beta-lactamases in South America. Clin. Microbiol. Infect. 14(Suppl 1):154-158.

von Baum, H., and R. Marre. 2005. Antimicrobial resistance of Escherichia coli and therapeutic implications. Int. J. Med. Microbiol. 295:503-511.

Wang, G., T. S. Whittam, C. M. Berg, and D. E. Berg. 1993. RAPD (arbitrary primer) PCR is more sensitive than multilocus enzyme electrophoresis for distinguishing related bacterial strains. Nucleic Acids Res. 21:5930-5933.

Wenz, J. R., G. M. Barrington, F. B. Garry, R. P. Ellis, and R. J. Magnuson. 2006. Escherichia coli isolates' serotypes, genotypes, and virulence genes and clinical coliform mastitis severity. J. Dairy Sci. 89:3408-3412.

Wilke, M. S., A. L. Lovering, and N. C. Strynadka. 2005. $\beta$-Lactam antibiotic resistance: A current structural perspective. Curr. Opin. Microbiol. 8:525-533.

Wu, G., M. Mafura, B. Carter, K. Lynch, M. F. Anjum, M. J. Woodward, and G. C. Pritchard. 2010. Genes associated with Escherichia coli isolates from calves with diarrhoea and/or septicaemia. Vet. Rec. 166:691-692. 